\title{
Hodgkin's lymphoma masquerading as vertebral osteomyelitis in a man with diabetes: a case report
}

\author{
Rachel A Bender Ignacio ${ }^{1}$, Anne Y Liu², Aliyah R Sohani ${ }^{3}$, Jatin M Vyas ${ }^{1,2^{*}}$
}

\begin{abstract}
Introduction: Infection and malignancy often have common characteristics which render the differential diagnosis for a prolonged fever difficult. Imaging and tissue biopsy are crucial in making a correct diagnosis, though differentiating between chronic osteomyelitis and malignancy is not always straightforward as they possess many overlapping features.

Case Presentation: A 52-year-old Caucasian man was treated with antibiotics for his diabetic foot infection after a superficial culture showed Staphylococcus aureus. He had persistent fevers for several weeks and later developed acute onset of back pain which was treated with several courses of antibiotics. Radiographic and pathological findings were atypical, and a diagnosis of Hodgkin's lymphoma was made 12 weeks later.

Conclusion: Clinicians should maintain a suspicion for Hodgkin's lymphoma or other occult malignancy when features of presumed osteomyelitis are atypical. Chronic vertebral osteomyelitis in particular often lacks features common to acute infectious disease processes, and the chronic lymphocytic infiltrates seen on histopathology have very similar features to Hodgkin's lymphoma, highlighting a similar inflammatory microenvironment sustained by both processes.
\end{abstract}

\section{Introduction}

Osteomyelitis of the spine is caused by direct instrumentation to the area, or contact with overlying softtissue infection, or by hematogenous seeding of the vertebrae. Risk factors for hematogenous vertebral osteomyelitis (HVO) include prolonged bacteremia, indwelling catheters, underlying diabetes, malignancy, or other immunocompromised states [1]. Several other disease processes can also present with vertebral lesions, including atypical infections and primary or metastatic malignancy. Hodgkin's lymphoma (HL) can present with asymptomatic mass lesions, B-symptoms or local symptoms in the location of the tumor bulk. It is most prevalent in young to middle-aged men. Lymphoma is not commonly found in the bone at presentation, and B-cell non-HLs are much more likely than HL to present in the bone. There is scant literature directly addressing bony lesions in $\mathrm{HL}$, especially in comparison to

\footnotetext{
* Correspondence: jvyas@partners.org

'Massachusetts General Hospital, Department of Medicine, Gray Building Room 740, 55 Fruit Street, Boston, MA 02114, USA
}

infectious disease processes [2]. This case demonstrates a patient who had the classic presentation and risk factors for HVO, but was ultimately found to have HL. The diagnostic difficulties, histology of biopsy samples, radiographic findings and disease similarities are discussed.

\section{Case Presentation}

A 52-year-old Caucasian man presented at an outside hospital with three days of fevers and a swollen, purpuric right foot. He had noted a necrotic-appearing ulcer on the plantar surface of his fifth digit one week previously. His past history was remarkable for diabetes mellitus type 2 (his last hemoglobin A1c [HbA1c] test was $7.0 \%$ ), his right great toe had been amputated secondary to infection in 2001; and he had a previous cigarette use of 60 pack-years. He had worked for the United States Forest Service, doing physical labor, often working in wet boots and with close contact to the feces of several species of forest animals. He had suffered a tick bite 6 months previously.

\section{()


On admission he was found to have a leukocytosis of 19,000 cells $/ \mathrm{mm}^{3}$ and mild normocytic anemia. The foot ulcer was superficially cultured and grew methicillinsensitive Staphylococcus aureus (MSSA). While hospitalized, he experienced chills, night sweats, nausea and vomiting. He was discharged and given cefazolin and metronidazole for a planned six-week course following normalization of his leukocyte count and resolution of systemic symptoms.

One month after discharge, he was re-admitted for evaluation of recurrent sweats, chills and weakness. His peripherally inserted central catheter (PICC) was removed, and the tip was cultured but yielded no growth. Surgical debridement of the fifth digit revealed no gross purulence, and broth from the deep tissue culture grew only Bacillus species. Vancomycin and piperacillin-tazobactam were substituted for the cefazolin and metronidazole regimen for a planned six weeks duration of therapy.

One week after admission, he had a new onset of lower back pain, prompting computed tomography (CT) and MRI scans of the spine which revealed diffuse bony lesions from T12 to L4. Microscopic analysis of a needle biopsy of the L4 lesion showed a mixed inflammatory infiltrate in a fibrotic background, interpreted as partially treated osteomyelitis (Figure 1a). Core needle biopsy of a right inguinal node demonstrated a dense mixed inflammatory infiltrate with rare large degenerated cells of uncertain significance (Figure 1b). Gram stain, acid-fast stain, and bacterial, mycobacterial and fungal cultures were negative from both biopsies. Antibiotics were discontinued and he was discharged.

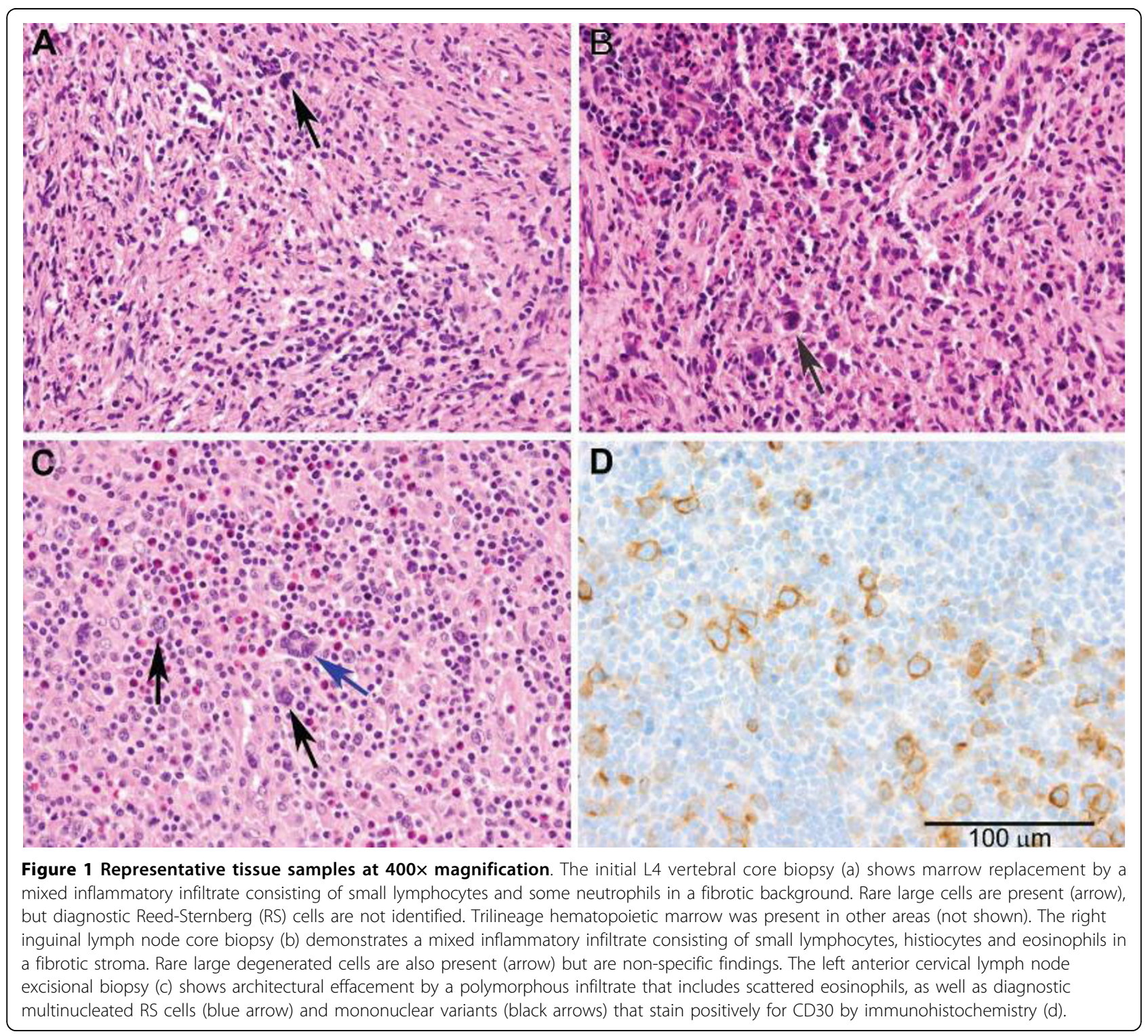


He was re-admitted one week later with recurrent systemic symptoms and a total leukocyte count of 20,000 cells $/ \mathrm{mm}^{3}$. Vancomycin and imipenem-cilastatin were started. Transesophageal echocardiogram revealed no valvular vegetations. A bone scan revealed multiple abnormal areas of uptake including the right foot, several ribs, scapula and both femurs. Blood cultures throughout these multiple hospitalizations did not recover any pathogenic organisms.

Upon transfer to our facility, he noted a $7 \mathrm{~kg}$ weight loss since the onset of symptoms, and he was fatigued but ambulatory. Examination was significant for a single $<2 \mathrm{~cm}$ soft, mobile, tender lymph node in the left anterior cervical chain and symmetric mild lymphadenopathy in both axillae and the groin. His right foot ulcer was well healed, though mild purpura and swelling remained over the third through fifth digits. His spine was not tender to palpation. Laboratory testing revealed a leukocyte count of 18,400 cells $/ \mathrm{mm}^{3}$ with $85 \%$ neutrophils and a platelet count of $404,000 / \mathrm{mm}^{3}$. C-reactive protein (CRP) was $83 \mathrm{mg} / \mathrm{L}$, erythrocyte sedimentation rate (ESR) $106 \mathrm{~mm} /$ hour, and alkaline phosphatase 461 U/L. Laboratory evaluations for tick-borne and endemic fungal infections were all negative, as were an antinuclear antibody (ANA) test, a rapid plasma regain (RPR) test, and a human immunodeficiency virus enzyme-linked immunosorbent assay (HIV ELISA). Intradermal purified protein derivative (PPD) did not elicit any induration. All antibiotics were discontinued, and the patient remained febrile at $38.3-39.3^{\circ} \mathrm{C}$ nightly with drenching sweats.

Ten sets of blood cultures were negative for bacteria, fungi and mycobacteria. CT scans of the chest, abdomen and pelvis revealed multiple small pulmonary nodules, bilateral small pleural effusions, a small pericardial effusion, two small calcified granulomas in the liver, and diffuse cervical, mediastinal, iliac and inguinal adenopathy (all $\leq 1.6 \mathrm{~cm}$ ). A repeat MRI of the spine confirmed multiple areas of $\mathrm{T} 1$ hypointensity and $\mathrm{T} 2$ enhancement throughout the cervical, thoracic and lumbar spine, sparing the intervertebral disks and the cord (Figure 2). Microscopic examination of a left cervical lymph node excisional biopsy and staging posterior iliac crest bone marrow biopsy revealed the presence of large atypical cells consistent with Reed-Sternberg (RS) cells and variants, and a diagnosis of Stage IV mixed-cellularity classical HL was made (Figures $1 \mathrm{c}$ and 1d). Positron emission tomography (PET)-CT was performed, displaying innumerable lesions in the axial spine and fluorodeoxyglucose (FDG)-avid nodes throughout innumerable lymphatic chains. Increased uptake was especially noted in the right lateral nasopharynx, without other solid

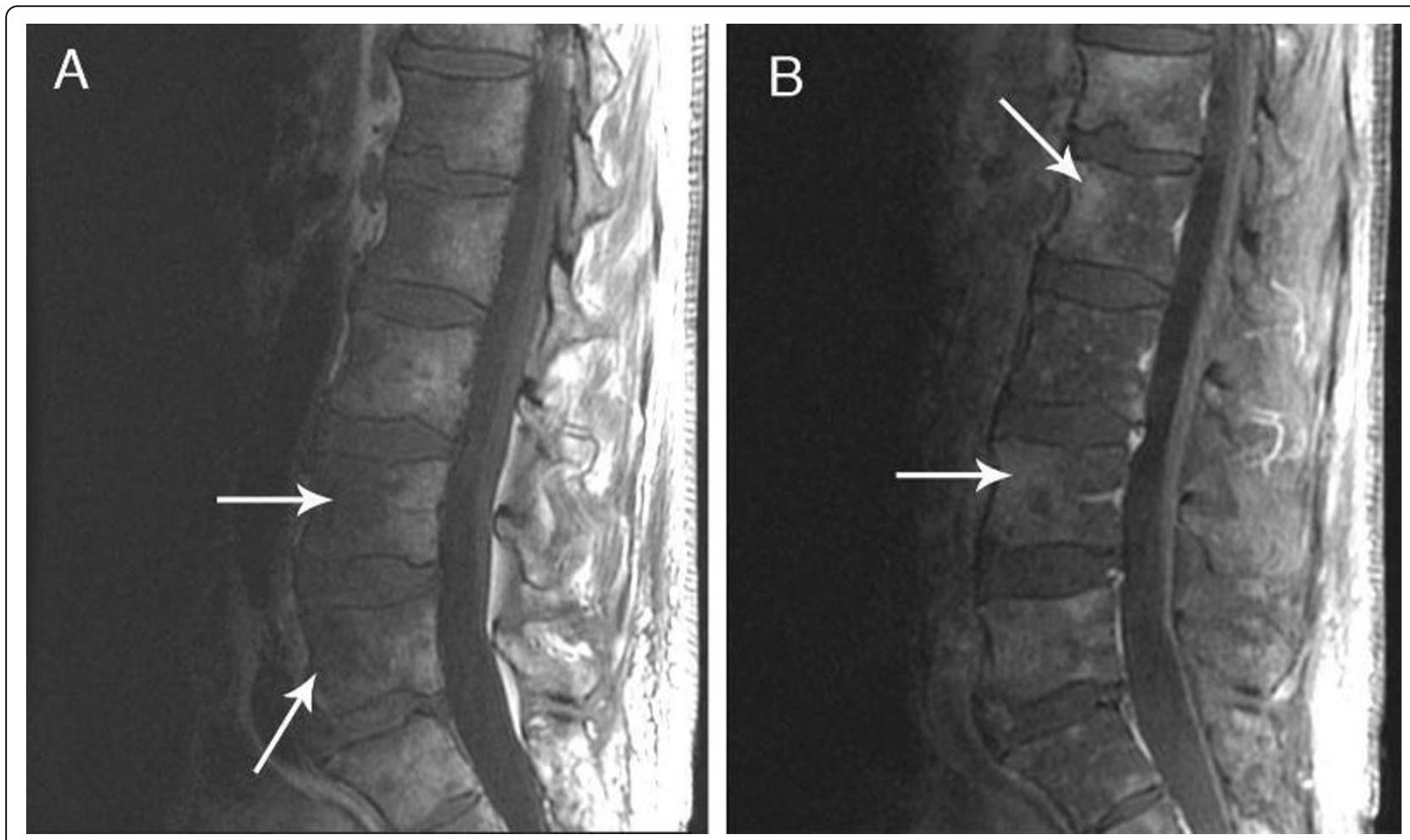

Figure 2 MRI of spine demonstrating multifocal hypointensities (arrows) sparing the intervertebral disks in T1-weighted images (a) The same lesions (arrows) appear hyperintense on T2-weighted images (b). No inflammation of the paraspinal muscles or abscess was identified. 


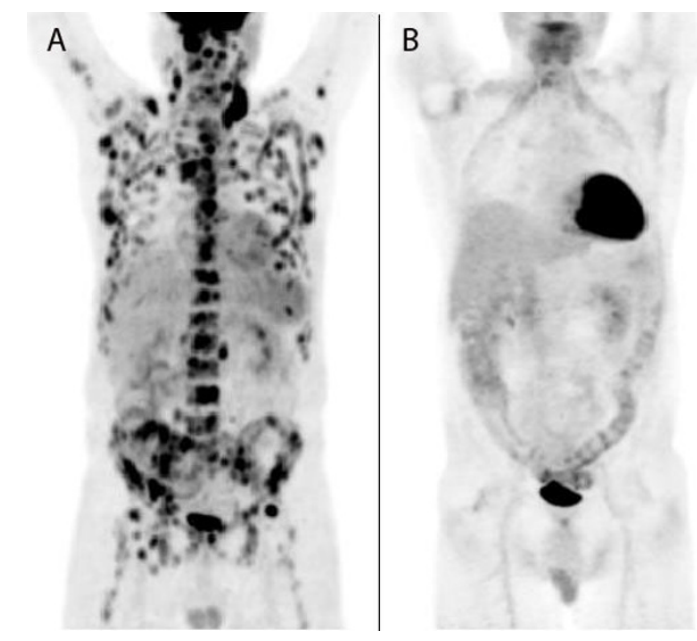

Figure 3 The scout film of the positron emission tomographycomputed tomography (PET-CT) scan performed prior to this first round of chemotherapy (a) demonstrates diffuse regions of uptake involving multiple ribs, multiple vertebral bodies, the pelvis, the sternum and the scapula. There is also increased fluorodeoxyglucose (FDG) uptake in multiple bilateral lymph node regions extending from the jugular, supraclavicular, mediastinal, retroperitoneal, pelvic and inguinal regions, consistent with Hodgkin's lymphoma. There is increased FDG uptake in the posterior and right lateral walls of the nasopharynx. About two months after his first round of chemotherapy, a repeat PET-CT scan (b) showed a marked interval decrease in the FDG-avid metastatic burden.

organ involvement. An escalated BE(A)COPP (bleomycin, etoposide, doxorubicin, cyclophosphamide, vincristine, procarbazine, and prednisone) regimen was initiated. Because of hematologic complications, our patient completed a course of modified Adriamycin [doxorubicin], bleomycin, vinblastine and dacarbazine (ABVD) chemotherapy and is in clinical and radiographic remission (Figure 3).

\section{Discussion}

Though it is common for malignancies and systemic infections to have overlapping features, several aspects of our case proved to be unique, ultimately delaying the diagnosis of $\mathrm{HL}$ in our patient. A progression from what became a chronic diabetic foot infection to vertebral osteomyelitis would have been logical. His underlying diabetes, partially treated infection, and ultimately discovered malignancy likewise placed him at significant risk for hematogenously seeded vertebral osteomyelitis [1]. Additionally, our patient denied 'B symptoms' prior to the appearance of his ulcer, and his leukocytosis and fevers temporarily resolved with initiation of each new antibiotic regimen, making the diagnosis of malignancy less likely. The presence of $S$. aureus in his necrotic diabetic foot ulcer concurrent with fevers then directed treatment of subsequent fevers exclusively towards persistent bacterial infection for several weeks.

Patients diagnosed with non-HIV associated HL are also diagnosed with twice as many infections in the 10 years prior to diagnosis as age-matched counterparts without malignancy, not including the year preceding diagnosis [3]. It is interesting to note that herpesviridae infections are more prominent in this population, presumably as a result of subtle immunological defects from their malignancy.

S. aureus accounts for nearly half of all cases of HVO and most commonly presents with back pain $(89 \%)$ and fever $(>60 \%)$ [4]. Initial characteristics of CT and MRI scans in our case raised suspicion for systemic involvement, though both a vertebral fine needle aspirate and core biopsy failed to confirm a diagnosis. Radiographic features of osseous HL and HVO are often indistinguishable [2,5]. Evidence of spondylodiscitis, though classic for infection, is not uniformly present in HVO, and is often absent without involvement of contiguous vertebrae. Imaging demonstrating paraspinal inflammation increases sensitivity and specificity for $\mathrm{HVO}$, but atypical organisms, such as mycobacteria, often lack paraspinal inflammation and are also more likely to demonstrate multi-level disease and skip lesions [6].

In $\mathrm{HL}$, the most common site of bony involvement is the spine, and multiple lesions at presentation are more common than a solitary lesion [7]. Radiographic features of our case made malignancy more likely, yet bone involvement at presentation of $\mathrm{HL}$ is quite uncommon, with only 33 cases being identified by biopsy in the last 70 years at the Mayo Clinic, with the majority of cases being primary osseous HL. When HL presented simultaneously in an osseous and a non-osseous site, $25 \%$ of such cases were initially misdiagnosed as osteomyelitis by histopathology [2]. Fine needle aspirates revealing lymphoma cells have a nearly perfect diagnostic accuracy, while those containing non-specific findings of osteomyelitis have insufficient positive or negative predictive value to confirm or exclude malignancy [8]. The infrequently encountered, often degenerated, malignant cells in our patient's initial biopsies illustrate the need for a high index of suspicion in such cases, and the importance of procuring additional tissue via excisional biopsy to confirm a diagnosis. The only site of solid organ involvement in our case proved to be within the nasopharynx, which is found to be the site of the primary lesion in less than $1 \%$ of all HL cases.

The diagnostic difficulties above highlight the similar molecular pathways of chronic inflammations seen in osteomyelitis and in HL. The microenvironment of HL is composed of a heterogeneous group of cells including $\mathrm{T}$ cells $(\mathrm{CD} 4+\mathrm{T}$ cells being the most prominent cell type), B cells, plasma cells, neutrophils, eosinophils and 
mast cells [9]. The prototypical RS cell represents only about $1 \%$ of the cells in the HL tumor. The expression of multiple cytokines by the RS cells appears to be critical in the development of the microenvironment and these other cell types appear to be required to sustain the viability of the RS cells [10]. It is interesting to note that RS cells survive in immunocompetent, but not immunodeficient, mice. RS cells secrete interleukin-8 (IL-8) which serves as a chemoattractant for neutrophils, and express multiple chemokine ligands including CCL5, CCL17 and CCL22, which attract certain T-cell subsets [9]. Osteomyelitis is more frequent in persons carrying the particular polymorphism of the Bax gene promoter also linked to the failure of these malignant cells to undergo apoptosis [11]. Increased serum levels of IL- 6 found in patients with active osteomyelitis play a causative role in decreased peripheral blood neutrophil apoptosis [12]. Both diseases produce a self-sustaining microenvironment that is reliant on competent host immunity to produce long-lived inflammatory cells generated by altered cell signaling.

\section{Conclusion}

Our case of Stage IV HL masquerading as osteomyelitis highlights the inherent difficulties in differentiating bone infection from malignant infiltration. Histopathological confirmation of HL only came after an inconclusive spinal biopsy and a lymph node core biopsy showing only rare atypical cells. Clinicians should maintain a suspicion for HL or other occult malignancy in patients with presumed osteomyelitis whose bony lesions appear atypical when analyzed by radiography or pathology, or in their response to treatment.

\section{Consent}

Written informed consent was obtained from the patient for publication of this case report and any accompanying images. A copy of the written consent is available for review by the Editor-in-Chief of this journal.

\section{Acknowledgements}

The authors thank Dr Jeremy Abramson for his thoughtful discussions. We would also like to thank Chris Bambacus, Stephen Conley and David Ignacio for their assistance with the figures.

\section{Author details \\ 1 Massachusetts General Hospital, Department of Medicine, Gray Building Room 740, 55 Fruit Street, Boston, MA 02114, USA. ²Massachusetts General Hospital, Division of Infectious Disease, Grey-Jackson Room 504, 55 Fruit Street, Boston, MA 02114, USA. ${ }^{3}$ Massachusetts General Hospital, Department of Pathology, Gray-Jackson Room 148-B, 55 Fruit Street, Boston, MA 02144, USA.}

\section{Authors' contributions}

$R A B \mid$ researched the topic, organized the paper, and prepared the radiographic images. $A Y L, R A B I$ and $J M V$ cared for the patient during his hospital admission. RABI and ARS prepared the histological samples for publication. All authors read and reviewed the final manuscript.

\section{Competing interests}

The authors declare that they have no competing interests.

Received: 5 June 2009 Accepted: 6 April 2010 Published: 6 April 2010

References

1. Isobe Z, Utsugi T, Ohyama Y, Miyazaki A, Ito H, Okuno S, Uchiyama T, Ohno T, Arai M, Tomono S, Kurabayashi M: Recurrent pyogenic vertebral osteomyelitis associated with type 2 diabetes mellitus. J Int Med Res 2001, 29:445-450.

2. Ostrowski ML, Inwards CY, Strickler JG, Witzig TE, Wenger DE, Unni KK: Osseous Hodgkin disease. Cancer 1999, 85:1166-1178.

3. Newton $R$, Crouch S, Ansell P, Simpson J, Willett EV, Smith A, Burton C, Jack A, Roman E: Hodgkin's lymphoma and infection: findings from a UK case-control study. Br J Cancer 2007, 97:1310-1314.

4. Sapico F, Montgomerie JZ: Vertebral osteomyelitis. Infect Dis Clin North Am 1990, 4:539-550.

5. Kayani I, Syed I, Saifuddin A, Green R, MacSweeney F: Vertebral osteomyelitis without disc involvement. Clin Radiol 2004, 59:881-891.

6. Ledermann HP, Schweitzer ME, Morrison WB, Carrino JA: MR imaging findings in spinal infections: rules or myths? Radiology 2003, 228:506-514.

7. Newcomer LN, Silverstein MB, Cadman EC, Farber LR, Bertino JR, Prosnitz LR: Bone involvement in Hodgkin's disease. Cancer 1982 49:338-342.

8. Rezanko T, Sucu HK, Akkalp A, Tunakan M, Sari A, Minoglu M, Bolat B: Is it possible to start the treatment based on immediate cytologic evaluation of core needle biopsy of the spinal lesions? Diagn Cytopathol 2008, 36:478-484.

9. Kuppers R: The biology of Hodgkin's lymphoma. Nat Rev Cancer 2009, 9:15-27.

10. Skinnider BF, Mak TW: The role of cytokines in classical Hodgkin lymphoma. Blood 2002, 99:4283-4297.

11. Ocana MG, Valle-Garay E, Montes AH, Meana A, Carton JA, Fierer J, Celada A, Asensi V: Bax gene G(-248)A promoter polymorphism is associated with increased lifespan of the neutrophils of patients with osteomyelitis. Genet Med 2007, 9:249-255.

12. Asensi V, Valle E, Meana A, Fierer J, Celada A, Alvarez V, Paz J, Coto E, Carton JA, Maradona JA, Dieguez A, Sarasua J, Ocana MG, Arribas JM: In vivo interleukin-6 protects neutrophils from apoptosis in osteomyelitis. Infect Immun 2004, 72:3823-3828.

doi:10.1186/1752-1947-4-102

Cite this article as: Bender Ignacio et al:: Hodgkin's lymphoma

masquerading as vertebral osteomyelitis in a man with diabetes: a case report. Journal of Medical Case Reports 2010 4:102.

\section{Submit your next manuscript to BioMed Central and take full advantage of:}

- Convenient online submission

- Thorough peer review

- No space constraints or color figure charges

- Immediate publication on acceptance

- Inclusion in PubMed, CAS, Scopus and Google Scholar

- Research which is freely available for redistribution

Submit your manuscript at www.biomedcentral.com/submit
C BioMed Central 